\title{
The theory of the spin-lattice relaxation of copper in a Tutton salt crystal
}

\author{
A. M. STONEHAM $\uparrow$ \\ H. H. Wills Physics Laboratory, University of Bristol \\ MS. received 17th August 1964
}

\begin{abstract}
A calculation of the relaxation of $\mathrm{Cu}^{2+}$ in potassium zinc Tutton salt has been made on a crystal field model. The magnitude and anisotropy predicted are in good agreement with experiment. The results imply that the tetragonal field splitting $\delta$ is about $7700 \mathrm{~cm}^{-1}$ and the cubic field splitting $\Delta_{0}$ about $8700 \mathrm{~cm}^{-1}$.
\end{abstract}

\section{Introduction}

Experimental and theoretical studies of the spin-lattice relaxation of magnetically dilute systems of transition series ions in non-magnetic host lattices suggest that the processes involved are not yet fully understood. One of the purposes of the present calculation was to see how well the commonly accepted relaxation mechanisms worked in one particular situation.

Cupric ions enter substitutionally for zinc in potassium zinc Tutton salt, and the experimental advantages of this system will be discussed in the following paper (Gill 1964). There is a further advantage that $\mathrm{Cu}^{2+}$, having an isolated Kramers doublet lowest, may shed some light on the two similar systems $\mathrm{Ti}^{3+}$ and $\mathrm{Co}^{2+}$, which relax at helium temperatures between three and five orders of magnitude faster than expected (Van Vleck 1940, Foglio and Pryce, to be published).

The Hamiltonian of $\mathrm{Cu}^{2+}$ in a static crystal field may be written

$$
\mathscr{H}_{\text {cu }}=\mathscr{H}_{0}+\mathscr{H}_{\text {cr }}+\mathscr{H}_{\text {so }}+\mathscr{H}_{\mathrm{z}}+\mathscr{H}_{\mathrm{I}}
$$

where the terms are in order of importance. The free-ion Hamiltonian $\mathscr{H}_{0}$ gives rise to a ${ }^{2} \mathrm{D}$ ground state. $\mathscr{H}_{\mathrm{Cr}}$ describes the interaction of the crystal with the ion, and will be taken to the crystal field approximation. It is assumed that $\mathscr{H}_{\mathrm{cr}}$ is entirely due to the distorted octahedron of water molecules surrounding the ion, whose effect is represented by an appropriate electrostatic potential. Overlap of the $3 \mathrm{~d}$ electron wave functions of the $\mathrm{Cu}^{2+}$ ion and the ligands will be neglected, as is the possibility of electron transfer between the ion and the ligands. The spin-orbit interaction $\mathscr{H}_{\mathrm{so}}$ is $\lambda \mathbf{L}$.S within the $3 \mathrm{~d}$ configuration for a hole in the $3 \mathrm{~d}$ shell described by $\mathbf{L}, \mathbf{S} . \mathscr{H}_{\mathrm{z}}$ is the electronic Zeeman energy. The nuclear Zeeman energy, together with terms arising from the interaction of the nuclear magnetic moment with the electronic moment, the anomalous s-electron contribution and quadripolar interactions, are contained in $\mathscr{H}_{\mathrm{r}}$. Although $\mathscr{H}_{I}$ introduces extra terms in the relaxation rate, these prove to be small and will not be discussed. 


\section{The $\mathrm{Cu}\left(\mathrm{H}_{2} \mathrm{O}\right)_{6}{ }^{2+}$ complex}

The cupric $3 \mathrm{~d}$ shell contains nine electrons, and can be treated as a hole in a closed $3 \mathrm{~d}^{10}$ shell. The orbitals for the hole are labelled $\epsilon, \theta, \xi, \eta$, $\zeta$, and transform under the cubic group as $x^{2}-y^{2}, 2 z^{2}-x^{2}-y^{2}, y z, x z$ and $x y$ respectively. In a static strictly cubic field, $\xi, \eta, \zeta$ are degenerate, as are $\epsilon, \theta$ which form the degenerate ground state. A lower energy configuration may be obtained by a tetragonal distortion of the complex of the six ligands and the $\mathrm{Cu}^{2+}$ ion. This follows from the theorem of Jahn and Teller, and is illustrated in figures 1 and 2 . The terms quadratic in the distortion arise from the usual forces which maintain the equilibrium of the crystal, and are the same for all the energy levels. The terms linear in the distortion are seen most clearly by subtracting off the common quadratic terms as in figure $2(b)$ and are the terms whose existence follows from the theorem cited.

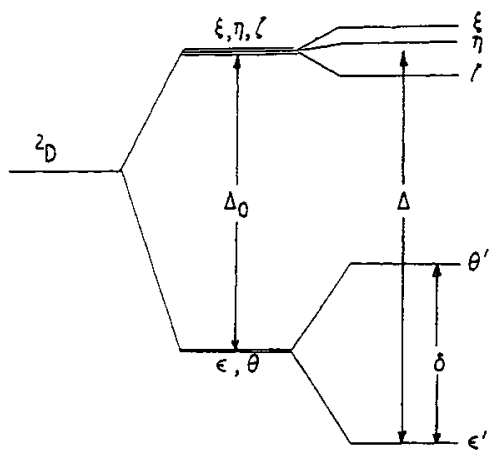

Figure 1. The energy levels of $\mathrm{Cu}^{2+}$ in crystal fields of cubic and lower symmetry.

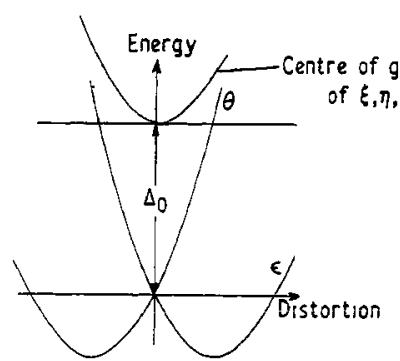

(a)

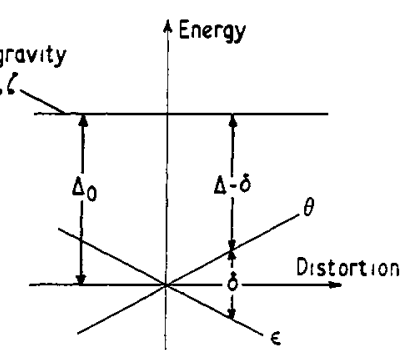

(b)

Figure 2. The effect of a tetragonal distortion on the energy levels of $\mathrm{Cu}^{2+}$. The distortion from an octahedral arrangement of ligands is positrve when two ligands move away from and four towards the $\mathrm{Cu}^{2+}$ ion. The curves for $\xi, \eta, \zeta$ lie close to that of their centre of gravity. In (b) a common quadratic term has been substracted from all energy levels.

In a rhombic field all degeneracy except Kramers' degeneracy is lost. The members of the orbital triplet $(\xi, \eta, \zeta)$ are still nearly degenerate in the case of dilute deuterated potassium copper Tutton salt (Bleaney, Bowers and Pryce 1955) and their splitting will be neglected in this calculation. The splitting of the orbital doublet $(\epsilon, \theta)$ by the tetragonal field is not altered by small rhombic components, so the result

$$
\Delta=\Delta_{0}+\frac{1}{2} \delta \text { or } \Delta_{0}=\Delta\left(1-\frac{1}{2 d}\right)
$$


which can be seen from figure $2(b)$ for the tetragonal case, is still valid. $\Delta, \Delta_{0}$ and $\delta$ are defined in the figure and $d=\Delta / \delta$. The rhombic term alters the wave functions of the doublet $\epsilon, \theta$, giving

$$
\begin{aligned}
|\epsilon\rangle \rightarrow\left|\epsilon^{\prime}\right\rangle & =|\epsilon\rangle \cos \alpha+|\theta\rangle \sin \alpha \\
|\theta\rangle \rightarrow\left|\theta^{\prime}\right\rangle & =|\theta\rangle \cos \alpha-|\epsilon\rangle \sin \alpha .
\end{aligned}
$$

$\alpha$ can be estimated from the $g$ values, as

$$
\begin{aligned}
& g_{x}-2 \simeq-\frac{2 \lambda}{\Delta}(\cos \alpha+\sqrt{3} \sin \alpha)^{2} \\
& g_{y}-2 \simeq-\frac{2 \lambda}{\Delta}(\cos \alpha-\sqrt{3} \sin \alpha)^{2} \\
& g_{z}-2 \simeq-\frac{8 \lambda}{\Delta} \cos ^{2} \alpha .
\end{aligned}
$$

(Abragam and Pryce 1951)

Using $g_{x}=2 \cdot 16, g_{y}=2.04$ and $g_{z}=2.42$, we find $\alpha=9^{\circ} 22^{\prime}$ and $\lambda / \Delta \simeq 0.054$. The average over all angles of $g$ is $2 \cdot 21$. The matrix elements of orbital angular momentum, given in table 1 , are zero within the sub-space $\epsilon, \theta$. Thus, as $\lambda / \Delta$ is so small, it is adequate to treat $\mathscr{H}_{\text {so }}$ and smaller terms to an appropriate order of perturbation theory, whereas $\mathscr{H}_{0}$ and $\mathscr{H}_{\mathrm{cr}}{ }^{0}$ must be diagonalized to the extent indicated above. $\mathscr{H}_{\mathrm{cr}}{ }^{0}$ is the value of $\mathscr{H}_{\mathrm{cr}}$ for the ligands in their static equilibrium positions.

$\mathscr{H}_{\mathrm{Cr}}$ is modulated by thermal and zero point vibrations, giving the spin-lattice interaction

$$
\mathscr{H}^{\prime}=\mathscr{H}_{\mathrm{Cr}}(\mathbf{r}, \mathbf{R})-\mathscr{H}_{\mathrm{Cr}}\left(\mathbf{r}, \mathbf{R}_{0}\right)
$$

where $\mathbf{R}$ represents the nuclear coordinates of the complex, $\mathbf{R}_{0}$ their static equilibrium values and $\mathbf{r}$ the coordinates of all the electrons of the $\mathrm{Cu}^{2+}$ ion. As $\mathbf{R}-\mathbf{R}_{0}$ will be small compared with $R$, an appropriate $\mathrm{Cu}^{2+}-\mathrm{H}_{2} \mathrm{O}$ separation, a Taylor expansion can be made:

$$
\mathscr{H}^{\prime}=\sum_{\mathrm{Cu}^{2+}} \sum_{\text {electrons }}\left\{\sum_{\alpha} W_{\alpha}\left(\mathbf{r}_{\jmath}\right) \frac{Q_{\alpha}}{R}+\sum_{\alpha, \beta} W_{\alpha \beta}\left(\boldsymbol{r}_{\jmath}\right) \frac{Q_{\alpha} Q_{\beta}}{R^{2}}+\ldots\right\}
$$

where the $Q_{\alpha}$ are the normal coordinates of the complex. $\mathscr{H}^{\prime}$ is invariant under operations of the symmetry group of the complex, including the inversion operator. Thus $W_{\alpha}$ has the same parity as $Q_{\alpha}$, and will have non-zero matrix elements within $3 \mathrm{~d}^{9}$ only if $Q_{\alpha}$ has even parity. The $Q_{\alpha}$ of odd parity can contribute pairwise in the second term of $\mathscr{H}^{\prime}$, but are negligible as the intermediate states are about $70000 \mathrm{~cm}^{-1}$ away.

The $W_{\alpha}$ and $Q_{\alpha}$ will be taken to be those for a complex with complete cubic symmetry. The $W_{\alpha} / R$ are then the $V_{\alpha}$ defined by Van Vleck (1939), and the $Q_{\alpha}$ are linear combinations of distortions of the complex which transform according to irreducible representations under the cubic group. The neglect of non-cubicity may be excused in several ways. Firstly, it is hard to improve on the approximation with the available information, for the environment of the $\mathrm{Cu}^{2+}$ ion is not well known; probably it consists of a rhombically distorted octahedron of water molecules whose dipole moments are not necessarily parallel to the octahedron axes. Secondly, other simplifications are needed in this calculation, such as the use later of the approximation of plane waves for the normal coordinates of the crystal. An exact solution for these for a crystal as complex as a Tutton salt is extremely difficult, yet it would be unrealistic to include non-cubicity 

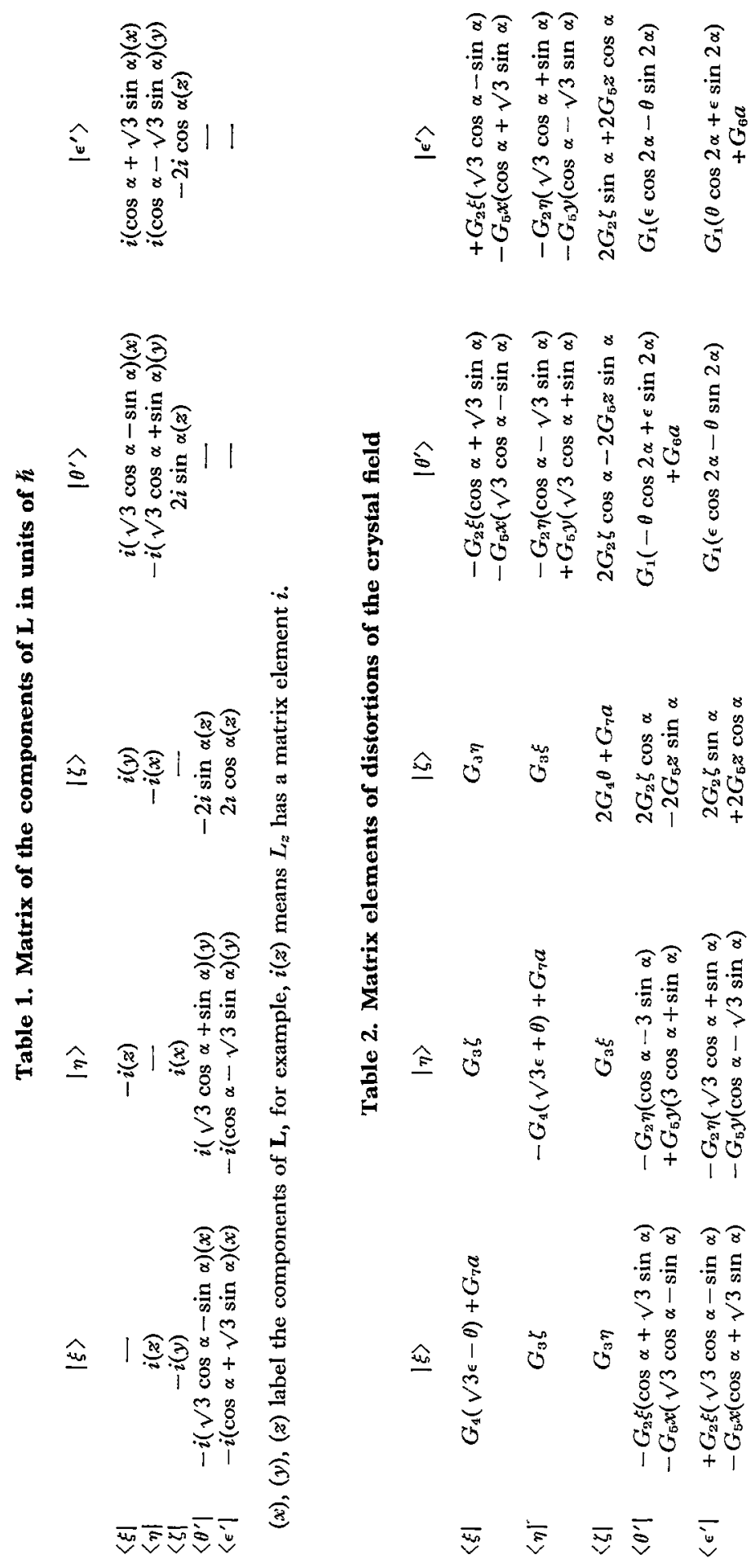
but to use this simplification. Thirdly, it will appear later that $Q_{2}$ is largely responsible for the relaxation. This only involves motion of the ligands in the plane normal to the tetragonal axis, and the configuration of these molecules is altered only in size by the static tetragonal distortion. The correct $W_{2}$ is that for the cubic case, using a value of $R$ appropriate to the ligands involved. As the way the $W_{\alpha}$ transforms under operations of the cubic group is known, their matrix elements between the orbitals $\epsilon, \theta, \xi, \eta, \zeta$, whose wave functions transform like those of $\mathrm{d}$ orbitals, are proportional to the elements of known matrices (Griffith 1961, $\S 8.7$ and A.20). The proportionality constants can be calculated and a transformation made to the orbitals which diagonalize $\mathscr{H}_{0}+\mathscr{H}_{\mathrm{cr}}$. The result appears as table 2 .

The $W_{\alpha}$ form bases for the representations $\mathrm{A}_{1}$ ( $\alpha=1$, breathing mode), $\mathrm{E}(\alpha=2,3$, pumping mode), $T_{1}\left(\alpha=19,20,21\right.$, rotations) and $T_{2}(\alpha=4,5,6$, shearing mode) of the cubic group. There are seven non-zero proportionality constants $G_{\alpha}$ corresponding to the elements of $\alpha=1$ and $\alpha=2,3$ in the $(\epsilon, \theta)$ sub-space of $\alpha=1, \alpha=2,3$ and $\alpha=4,5,6$ within the $(\xi, \eta, \zeta)$ sub-space, and of $\alpha=4,5,6$ and $\alpha=19,20$, 21 between the two sub-spaces. They can be expressed, in the approximation that the ligands give an isotropic field of force $\mu / x^{2}$ at distance $x$ from the ligand, in terms of $\mu, R, e$ (the magnitude of the electronic charge) and $\left\langle r^{n}\right\rangle$, the expectation value of the $n$th power of the mean distance of a $3 \mathrm{~d}$ electron from the nucleus. $\left\langle r^{n}\right\rangle$ is assumed to be the same for all $\mathrm{d}$ orbitals. To this approximation,

where

$$
\Delta_{0}=\Delta\left(1-\frac{\delta}{2 \Delta}\right)=\frac{25}{3} \frac{e \mu}{R^{2}} \rho_{4}
$$

$$
\begin{aligned}
& \rho_{n}=\left\langle r^{n}\right\rangle / R^{n}, \quad \text { and if } \gamma_{\imath}=G_{2} / G_{1} \\
& G_{1}=-\frac{3 \sqrt{ } 3}{7} \Delta_{0}\left(1+\frac{24 \rho_{2}}{25 \rho_{4}}\right) \\
& \gamma_{2}=-\frac{1}{5}\left(1+\frac{8 \rho_{2}}{5 \rho_{4}}\right)\left(1+\frac{24 \rho_{2}}{25 \rho_{4}}\right)^{-1} \\
& \gamma_{3}=-\frac{4}{5 \sqrt{ } 3}\left(1-\frac{6 \rho_{2}}{5 \rho_{4}}\right)\left(1+\frac{24 \rho_{2}}{25 \rho_{4}}\right)^{-1} \\
& \gamma_{4}=-\frac{2}{3}\left(1-\frac{18 \rho_{2}}{25 \rho_{4}}\right)\left(1+\frac{24 \rho_{2}}{25 \rho_{4}}\right)^{-1} \\
& \gamma_{5}=-\frac{21}{25 \sqrt{ } 3}\left(1+\frac{24 \rho_{2}}{25 \rho_{4}}\right)^{-1} \\
& \gamma_{6}=\frac{7}{15 \sqrt{ } 2}\left(1+\frac{24 \rho_{2}}{25 \rho_{4}}\right)^{-1} \\
& \gamma_{7}=-\frac{14}{45 \sqrt{ } 2}\left(1+\frac{24 \rho_{2}}{25 \rho_{4}}\right)^{-1} .
\end{aligned}
$$

Using a value for $\rho_{2} / \rho_{4}$ obtained from the calculation for $\mathrm{Cu}^{1+}$ by Hartree and Hartree (1936) and $R=2.05 \AA$,

$$
\begin{array}{cc}
G_{1}=-3.40 \Delta_{0} \\
\gamma_{2}=-0.304 & \gamma_{5}=-0.106 \\
\gamma_{3}=+0.350 & \gamma_{6}=+0.072 \\
\gamma_{4}=+0.318 & \gamma_{7}=-0.048 .
\end{array}
$$


$\gamma_{6}, \gamma_{7}$ will be dropped from now on. Their contribution to the relaxation rate is a further order of magnitude smaller than (3) suggests, for equation (4) shows that this contribution is reduced by the fifth power of the ratio of the velocities of transverse to longitudinal waves.

The distortions $Q_{\alpha}$ of the complex can be related to the normal coordinates of the crystal. It is necessary to make a number of approximations, in particular that the crystal may be assumed isotropic, that the mean square displacements are independent of the site in the unit cell and that the long wave limit may be used. These should be reasonable at temperatures much less than the Debye temperature. The isotropy assumption means that the acoustic phonons (which are almost exclusively responsible for relaxation at low temperatures) can be described in terms of one longitudinal and two transverse modes, and together with the homogene1ty assumption means that the normal coordinates of the crystal can be taken as standing plane waves. From the long wave limit it follows that, for phonons of wave vector $\mathbf{f}$ and polarization $s,|\mathbf{f}| R \ll 1$ and that $\omega_{i s}=|\mathbf{f}| v_{s}$. Here $\omega_{\mathrm{fs}}$ is the corresponding angular frequency and $v_{s}$ the relevant velocity of sound. Then

$$
\frac{Q_{\alpha}}{R}=\sum_{\mathbf{f}, s}\left(\frac{\hbar \omega_{\mathrm{fs}}}{2 M_{\mathrm{c}} v_{s}^{2}}\right)^{1 / 2} \psi_{\alpha}(\mathbf{f}, s)\left(a_{\mathrm{fs}}+a_{\mathrm{fs}}{ }^{+}\right) \sin \phi_{\mathbf{f} s}
$$

where $M_{\mathrm{c}}$ is the crystal mass, and $a_{\mathrm{fs}}, a_{\mathrm{fs}}{ }^{+}$phonon annihilation and creation operators, with matrix elements

$$
\left\langle n_{\mathrm{fs}}\left|a_{\mathrm{fs}}\right| n_{\mathrm{fs}}+1\right\rangle=\left\langle n_{\mathrm{fs}}+1\left|a_{\mathrm{fs}}{ }^{+}\right| n_{\mathrm{fs}}\right\rangle=\left(n_{\mathrm{fs}}+1\right)^{1 / 2} .
$$

$n_{\mathrm{fs}}$ is the phonon occupation number of the mode $\mathbf{f}, s$ in thermal equilibrium and is $\left\{\exp \left(\hbar \omega_{\mathrm{fs}} / k T\right)-1\right\}^{-1} . \phi_{\mathrm{fs}}$ is a phase which appears because the nodes of $(\boldsymbol{f}, s)$ have some distribution with respect to the $\mathrm{Cu}^{2+}$ ion. For a random distribution the expectation value of $\sin ^{2} \phi_{\mathrm{fs}}$ is $\frac{1}{2}$. The $\psi_{\alpha}$ transform under the cubic group in the same way as the $Q_{\alpha}$ and are functions of the phonon polarization vector with components $\left(X_{\mathrm{fs}}, Y_{\mathrm{fs}}, Z_{\mathrm{fs}}\right)$ and the unit wave vector $\mathbf{f} / \mathbf{f} \mid$ with components $(L, M, N)$. For each phonon $(\mathbf{f}, s)$

$$
\begin{array}{ll}
\psi_{2}=X L-M Y & \psi_{3}=\frac{1}{\sqrt{ } 3}(2 Z N-X L-Y M) \\
\psi_{4}=Y N+Z M & \psi_{19}=Y N-Z M \\
\psi_{5}=Z L+X N & \psi_{20}=Z L-X N \\
\psi_{6}=X M+Y L & \psi_{21}=X M-Y L .
\end{array}
$$

For later use we need the two summations

$$
\begin{aligned}
& \sum_{\mathrm{I}}(\alpha, \beta)=\sum_{s, I / /} \frac{\psi_{\alpha}^{*} \psi_{\beta}}{v_{s}^{5}} \\
& \sum_{\mathrm{II}}(\alpha, \beta, \gamma, \delta)=\sum_{\substack{s, s^{\prime} \\
f^{\prime} / f^{\prime}, \text { Iff }}} \frac{\left(\psi_{\alpha} \psi_{\beta}^{\prime}-\psi_{\alpha}^{\prime} \psi_{\beta}\right) *\left(\psi_{\gamma} \psi_{\delta}^{\prime}-\psi_{\gamma}{ }^{\prime} \psi_{\delta}\right)}{v_{s}^{5} v_{s^{\prime}}{ }^{5}}
\end{aligned}
$$

where

$$
|\mathbf{f}|=f \text { and } \psi_{\alpha}=\psi_{\alpha}(\mathbf{f}, s), \psi_{\alpha}{ }^{\prime}=\psi_{\alpha}\left(\mathbf{f}^{\prime}, s^{\prime}\right) .
$$

By transforming $\Sigma_{f / f}$ into an integral $\int_{4 \tau} d \Omega_{\mathrm{f}}$ and using the transformation properties 
of the $\psi_{\alpha}$ under the cubic group, we obtain

$$
\begin{aligned}
\sum_{I}(\alpha, \beta)= & \delta_{\alpha \beta} \frac{4 \pi}{15}\left(\frac{4}{v_{1}^{5}}+\frac{6}{v_{t}^{5}}\right), \alpha=2,3,4,5,6 \\
& \delta_{\alpha \beta} \frac{4 \pi}{15}\left(\frac{10}{v_{t}^{5}}\right), \quad \alpha=19,20,21 \\
& \delta_{\alpha \beta} \frac{4 \pi}{15}\left(\frac{10}{v_{1}^{5}}\right), \quad \alpha=1 \\
\sum_{\mathrm{II}}(\alpha, \beta, \alpha, \beta)= & 2\left(1-\delta_{\alpha \beta}\right) \sum_{I}(\alpha, \alpha) \sum_{I}(\beta, \beta)
\end{aligned}
$$

Apart from some obvious permutations, all other $\Sigma_{\mathrm{II}}(\alpha, \beta, \gamma, \delta)$ are zero.

The different velocity factor for rotations can be simply included by multiplying $G_{5}$ by $\left(5 / v_{t}^{5}\right)\left(2 / v_{1}^{5}+3 / v_{t}^{5}\right)^{-1}$. For $v_{1} / v_{t}=1 \cdot 5, \gamma_{5}$ becomes -0.131 , and it is this which is used in all subsequent stages. As the contribution from rotations is not large, the estimate of $v_{1} / v_{t}$ is not of critical importance.

\section{Transition probabilities}

We write the Hamiltonian for a crystal containing a single $\mathrm{Cu}^{2+}$ ion

$$
\mathscr{H}=\mathscr{H}_{\text {Cu }}+\mathscr{H}_{\text {latt1ce }}+\mathscr{H}^{\prime}
$$

where

$$
\mathscr{H}_{\text {lattice }}=\sum_{\mathrm{f}, \mathrm{s}} \hbar \omega_{\mathrm{fs}}\left(a_{\mathrm{fs}}{ }^{+} a_{\mathrm{fs}}+\frac{1}{2}\right)
$$

in the harmonic approximation, and, omitting $\mathscr{H}_{I}$ which proves to be unimportant,

$$
\mathscr{H}_{\mathrm{Cu}}=\left(\mathscr{H}_{0}+\mathscr{H}_{\mathrm{Cr}}{ }^{0}+2 \beta \mathbf{S} . \mathbf{H}\right)+(\lambda \mathbf{L} . \mathbf{S}+\beta \mathbf{L} . \mathbf{H})=\mathscr{H}^{\mathrm{A}}+P .
$$

Mattuck and Strandberg's method (1960, to be referred to as MS) is adopted. Like the method of Van Vleck (1940), $\mathscr{H}_{\text {lattice }}$ and $\mathscr{H}^{\mathrm{A}}$ are treated exactly, and $\mathscr{H}^{\prime}$ as a perturbation, but whereas Van Vleck also treats $P$ as a perturbation, $P$ is diagonalized in MS to an appropriate order by Lowdin's method. These two cases differ least when $P$ (which has no diagonal elements between eigenstates of $\mathscr{H}^{\mathrm{A}}$ ) is small compared with the separation of the ground states from the nearest excited states to which $P$ connects it. Here $P \sim \lambda<1000 \mathrm{~cm}^{-1}$ is much less than $\Delta>10^{4} \mathrm{~cm}^{-1}$. For the Raman process, the important terms are easier to pick out in the Van Vleck case, and this helps the selection of the important terms for the approach of MS.

The excited electronic states of the complex are $10^{3}$ to $10^{4} \mathrm{~cm}^{-1}$ above the ground state, so at most temperatures only the ground $\left(\epsilon^{\prime}\right)$ Kramers doublet is populated. It is therefore convenient to write a Hamiltonian which gives the transition probability for the direct or the Raman process in terms of $a_{f s}, a_{f s}{ }^{+}$and spin operators which operate between the eigenstates of a spin Hamiltonian of effective spin $\frac{1}{2}$ describing the behaviour of the lowest Kramers doublet.

Let the doublet be $|+\rangle,|\rightarrow\rangle$ and $E_{+}-E_{-}=\hbar \omega_{0}$. Then

$$
\begin{aligned}
\mathscr{H}_{\text {direct }}=\sum_{\substack{f, s \\
\imath>j}}\left(\frac{\hbar \omega_{\mathrm{fs}}}{2 M_{\mathrm{o}} v_{s}^{2}}\right)^{1 / 2} \psi_{\alpha}(\mathbf{f} s)\left(a_{\mathrm{fs}}+{a_{\mathrm{fs}}}^{+}\right) \sin \left(\phi_{\mathrm{f} s}\right) 2 \lambda \beta H \\
\quad \times\left\{\mathscr{L}_{\imath}{ }^{\alpha} g_{k k^{\prime}} S_{\imath}+\mathscr{L}_{\imath \jmath}{ }^{\alpha}\left(S_{\imath} l_{3 z}+S_{\jmath} l_{\imath z}\right)\right\}
\end{aligned}
$$




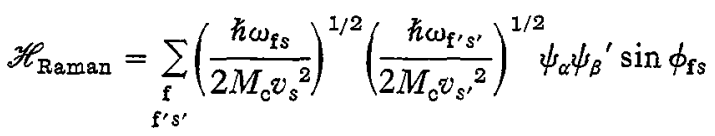

$$
\begin{aligned}
& \alpha \beta \\
& \times \sin \phi_{\mathbf{f}^{\prime} s^{\prime}}\left(a_{\mathrm{fs}}+a_{\mathrm{fs}}{ }^{+}\right)\left(a_{\mathrm{f}^{\prime} \mathrm{s}^{\prime}}+a_{\mathrm{f}^{\prime} \mathrm{s}^{\prime}}{ }^{+}\right) \mathscr{G}_{2 \alpha \beta}^{\mathrm{ff} \mathrm{fs}^{\prime}} S_{2} .
\end{aligned}
$$

The tensors $\mathscr{L}_{i}^{\alpha}$ and $\mathscr{L}_{i 3}{ }^{\alpha}$ are defined in equation (45) of MS, and $\mathscr{G}_{2, \alpha \beta}$ is the first term in MS, equation (57). The Raman process has contributions from the first term in $\mathscr{H}^{\prime}$ taken to second order, or the second term to first order. These terms are proportional to factors of order $\left(W^{2} / \delta\right) f_{1}, W f_{2}$, where $W \sim W_{\alpha} \sim W_{\alpha \beta}$ and $f_{1} \sim k T, f_{2} \sim \hbar \omega_{0}=g \beta H$ appear because the contributions to the Raman process vanish in the limit of zero $f_{1}, f_{2}$ respectively. At all temperatures of interest the first term dominates, and the other will not be discussed further. The spin components $S_{\imath}$, referred to the axes of the complex, are related to the spin components $\sigma_{\imath}$, referred to axes in which the $z$ axis is parallel to the applied magnetic field of magnitude $H$, by

$$
S_{\imath}=\sum_{3} l_{\imath j} \sigma_{j}
$$

which also defines the $l_{\imath \jmath}$. For simplicity we can write

$$
\begin{aligned}
\mathscr{H}_{\text {direct }} & =F_{x} S_{x}+F_{y} S_{y}+F_{z} S_{z}=\Phi_{x} \sigma_{x}+\Phi_{y} \sigma_{y}+\Phi_{z} \sigma_{z} \\
\mathscr{H}_{\text {Raman }} & =P_{x} S_{x}+P_{y} S_{y}+P_{z} S_{z}=\left\{\frac{1}{2} \sum_{\jmath} P_{\jmath}\left(l_{3 x}-i l_{y y}\right)\right\} \sigma_{+} \\
& +\left\{\frac{1}{2} \sum_{\jmath} P_{\jmath}\left(l_{\partial x}+i l_{y y}\right)\right\} \sigma_{-}+\left\{\sum_{\jmath} P_{\jmath} l_{\jmath z}\right\} \sigma_{z} .
\end{aligned}
$$

The transformation of coordinates is simpler in the Raman case as the $P_{\jmath}$, unlike the $F_{\eta}$, do not involve the $l_{\imath \vartheta}$. Replacing the summations over phonons by integrals over a sphere containing the same number of phonons as the first Brillouin zone, the direct process transition probability is

$$
W_{+\rightarrow-}^{\mathrm{a} 1 \mathrm{rect}}=\frac{1}{4} \frac{2 \pi}{\hbar^{2}} \frac{V}{8 \pi^{3}} \sum_{s} \int d f f^{2} \int d \Omega_{\mathrm{f}}\left|\left\langle n_{\mathrm{f} s}+1\left|\Phi_{x}-i \Phi_{y}\right| n_{\mathrm{f} s}\right\rangle\right|^{2} \delta\left(\omega_{0}-\omega_{\mathrm{f} s}\right)
$$

where $V$ is the volume of the crystal. In detail, the relevant integrals over angles are given in (4). The relaxation time $\tau_{\text {durect }}$ is equal to $\left(W_{+\rightarrow-}^{\text {direct }}+W_{-\rightarrow+}^{\text {direct }}\right)^{-1}$ and is given by

$$
\frac{1}{\tau_{\mathrm{d} \text { Irect }}}=\frac{1}{3 \pi} \frac{\omega_{0}^{4}}{g^{2}} k T \frac{1}{\rho}\left(\frac{2}{5 v_{1}^{5}}+\frac{3}{5 v_{t}^{5}}\right)\left(\frac{\lambda}{\Delta}\right)^{2}\left(\frac{G_{1}}{\Delta}\right)^{2} \sum_{\alpha} \theta_{\alpha}
$$

The $\theta_{\alpha}$ are complicated functions of the $l_{\imath \jmath}, \Delta / \delta$, the rhombicity and the $\gamma_{2}$. The approximation

$$
2 n_{\omega_{0}}+1=\operatorname{coth} \frac{\hbar \omega_{0}}{2 k T} \simeq \frac{2 k T}{\hbar \omega_{0}}
$$

has been used, as $\hbar \omega_{0} / 2 k T<1 / 8$ for all the experiments. As $\omega_{0}=g \beta H / \hbar$, the direct process relaxation time is proportional to $H^{-4} T^{-1}$. Similarly

$$
\begin{gathered}
W_{+\rightarrow-}^{\text {Raman }}=\frac{1}{4} \frac{2 \pi}{\hbar^{2}}\left(\frac{V}{8 \pi^{3}}\right)^{2} \sum_{s, s^{\prime}} \iint d f d f^{\prime} f^{2} f^{\prime 2} \delta\left(\omega^{\prime}-\omega+\omega_{0}\right) \int d \Omega_{f} d \Omega_{f^{\prime}} \\
\times\left|\left\langle\left.\begin{array}{l}
n_{\mathrm{f} s}-1 \\
n_{f^{\prime} s^{\prime}}+1
\end{array}\right|^{\frac{1}{2}} \sum_{\jmath} P_{\jmath}\left(l_{j x}-i l_{\jmath y}\right) \mid \begin{array}{l}
n_{f s} \\
n_{f^{\prime} s^{\prime}}
\end{array}\right\rangle\right|^{2} .
\end{gathered}
$$


There are no cross terms in $P_{2} P_{y \neq 2}$ because none of the factors $\psi_{\alpha}^{\prime} \psi_{\beta}-\psi_{\alpha} \psi_{\beta}{ }^{\prime}$ occurs for more than one value of $i$. Ultimately

$$
\begin{aligned}
\frac{1}{\tau_{\text {Raman }}}=2 \frac{\hbar^{2}}{2 \pi^{3}}( & \left.\frac{G_{1}}{\Delta}\right)^{4}\left(\frac{\lambda}{\Delta}\right)^{2} \frac{1}{\rho^{2}}\left(\frac{2}{5 v_{1}^{5}}+\frac{3}{5 v_{t}^{5}}\right)^{2} 8 !\left(\frac{k T}{\hbar}\right)^{9} \Gamma\left(\frac{\Theta_{D}}{T}\right) \\
& \times\left\{\left(W_{y}+W_{z}\right)-\left(W_{y}-W_{x}\right) m^{2}+\left(W_{x}-W_{z}\right) n^{2}\right\}
\end{aligned}
$$

where

$$
\Gamma\left(x_{\mathrm{D}}\right)=\frac{1}{8 !} \int_{0}^{x_{\mathrm{D}}} \frac{d x\left(x^{8} \mathrm{e}^{x}\right)}{\left(\mathrm{e}^{x}-1\right)^{2}}
$$

tends to unity as $x_{\mathrm{D}}$ tends to infinity. $\Theta_{\mathrm{D}}$ is the Debye temperature and $\Gamma\left(x_{\mathrm{D}}\right)$ has been given by Ziman (1954). The $W_{2}$ represent the contribution of the corresponding $P_{2}$ and $(l, m, n)$ are the direction cosines of the magnetic field with respect to the axes of the complex. In this case $W_{x} \neq W_{y} \neq W_{z}$ so that the relaxation is anisotropic, but independent of the magnitude of the applied field. This contrasts with the case of a cubic environment when $W_{x}=W_{y}=W_{z}$, and the Raman process is independent of the direction of the applied field. The $W_{2}$ are complicated functions of $\Delta / \delta$, the $\gamma_{\imath}$ and the rhombicity. Hill and Smith (1953) have measured the specific heat of $\left(\mathrm{NH}_{4}\right) \mathrm{Zn}$ Tutton salt, from which one can derive $v_{N}$, where $v_{N}{ }^{-3}=\left(\frac{1}{3} v_{1}{ }^{-3}+\frac{2}{3} v_{t}{ }^{-3}\right)$. In calculating $\bar{v}_{K}{ }^{-5}=\frac{2}{5} v_{1}{ }^{-5}+\frac{3}{5} v_{t}{ }^{-5}$ for $(\mathrm{K}) \mathrm{Zn}$ Tutton salt, it is assumed that $\bar{v}_{\mathrm{K}}=v_{\mathrm{N}}$. This should not lead to a large error and gives $\bar{v}_{\mathrm{K}}^{5}=1 \cdot 0 \times 10^{27} \mathrm{~cm}^{5} \mathrm{sec}^{-5}$. Tutton (1916) quotes $\rho_{\mathrm{KZn}}=2 \cdot 246$. The remaining factors in (5) and (6) are $\lambda / \Delta$ which was derived from the $g$ values in (2), and $G_{1} / \Delta=\left(G_{1} / \Delta_{0}\right)(1-1 / 2 d)$ which is given in (3). It is not necessary to know $\lambda, \Delta_{0}$ or $G_{1}$ separately at any stage, which is very convenient in view of the lack of optical data. The one unknown parameter is $d=\Delta / \delta$, and both relaxation rates can be written in terms of this. $\omega_{0}$ is $0.9455 \times 2 \pi \times 10^{10} \mathrm{rad} \mathrm{sec}^{-1}$.

$$
\begin{aligned}
& \frac{1}{T_{\tau_{\text {direct }}}}=0.72 \times 10^{-3}\left(\frac{2 \cdot 21}{g}\right)^{2}\left(1-\frac{1}{2 d}\right)^{2}\left\{A+B n^{2}+C n^{4}+D\left(l^{4}+m^{4}+n^{4}\right)\right. \\
& \left.+E\left(l^{2}-m^{2}\right)+F n^{2}\left(l^{2}-m^{2}\right)\right\} \quad \operatorname{deg}^{-1} \sec ^{-1} . \\
& A=9 \cdot 95 d^{2}+7 \cdot 90 d+31 \cdot 24 \\
& B=-\left(8.62 d^{2}+6.90 d+80.02\right) \\
& C=8 \cdot 62 d^{2}+6 \cdot 90 d+79.91 \\
& E=+7 \cdot 72 \\
& D=-\left(9.95 d^{2}+7.90 d+0.99\right) \\
& F=15 \cdot 82 d^{2}+10 \cdot 86 d-35 \cdot 0 \\
& \frac{1}{T^{9} \tau_{\text {Raman }}}=2 \cdot 15 \times 10^{-8}\left(T-U m^{2}+V n^{2}\right)\left(1-\frac{1}{2 d}\right)^{4} \Gamma\left(x_{\mathrm{D}}\right) \\
& T=3.017 d^{4}+2 \cdot 677 d^{3}+1.783 d^{2}+0.512 d+0.981 \\
& U=0.200 d^{4}+0.863 d^{3}+0.411 d^{2}+0.048 d-0.181 \\
& V=0 \cdot 247 d^{4}+1 \cdot 946 d^{3}+1 \cdot 118 d^{2}+0 \cdot 378 d-0.532 \text {. }
\end{aligned}
$$

When the magnetic field is parallel to one of the axes of the complex (one of $l, m, n=1$ ), the dependence of the direct process on $d$ disappears except for $(1-1 / 2 d)^{2}$, where the terms in $d$ correspond to energy denominators $\delta=E_{\theta^{\prime}}-E_{\epsilon^{\prime}}$ rather than $\Delta$. In the sub-space $\left|\epsilon^{\prime}\right\rangle,\left|\theta^{\prime}\right\rangle \mathbf{L}$ has zero elements and there are only non-zero elements of $Q_{2}$ and $Q_{3}$, which transform under the cubic group like $x^{2}-y^{2}, 2 z^{2}-x^{2}-y^{2}$. As $\mathscr{H}^{\prime}$, which is linear in spin and magnetic field components, is invariant under these transformations, it will contain terms

$$
A_{\epsilon} Q_{2}\left(S_{x} H_{x}-S_{y} H_{y}\right)+A_{\theta} Q_{3}\left(2 \dot{S}_{z} H_{z}-S_{x} H_{x}-S_{y} H_{y}\right)
$$


where the axes are those of the complex. When $\boldsymbol{H}$ is parallel to one of these axes, the only non-zero terms are proportional to $H S_{\imath} \equiv H \sigma_{z}$. This has no off-diagonal elements, so the contributions of $Q_{2}, Q_{3}$, and hence the terms in $d$, will vanish for these orientations.

\section{Discussion of experimental results}

A detailed comparison of equations ( 7 ) and (8) with experiment is given in the following paper. The Tutton salts contain two types of $\mathrm{Cu}^{2+}$ site, which are related by a translation plus a reflection and whose tetragonal axes are nearly perpendicular. As, for most of the orientations considered their magnetic resonance lines overlap, it is expected that cross relaxation will be important. By assuming that the cross relaxation occurs much more rapidly than the spin-lattice relaxation and conserves the total Zeeman energy, rather good agreement results. The direct process relaxation rate when the magnetic field is parallel to the axes of the complex enables one to estimate the accuracy of the terms in (7) which involve $d$ only in the factor $(1-1 / 2 d)^{2}$. The predicted rate is about $0.67(1-1 / 2 d)^{2}$ of that observed, which is very reasonable considering the assumptions made, particularly those concerning the lattice system. A change in velocity of sound of about $25 \%$ would correct the terms, and would probably correspond to the inhomogeneity of the lattice. Correspondingly, the Raman process was corrected by a factor $(0.67)^{2} \times(1-1 / 2 d)^{4}$ before being used to estimate $d$. The question of cross relaxation is not so clear-cut here, as the cross-relaxation rate is temperature independent and will be more important at low temperatures. It appears that the estimate of $d=1.64$ is not sensitive to the extent of the cross relaxation, and so this value was used to predict the orientation dependence of the direct process.

This estimate of $d$ is compatible with optical data given by Griffith (1961). In aqueous solution $\Delta$ appears to be $12600 \mathrm{~cm}^{-1}$, which, with $d=1.64$ and equation (1), gives $\delta \sim 7700 \mathrm{~cm}^{-1}$ and $\Delta_{0} \sim 8700 \mathrm{~cm}^{-1}$. This is consistent with the values for hexaquo-complexes of adjacent divalent ions as $\Delta_{0} \sim 7900 \mathrm{~cm}^{-1}$ for $\mathrm{Co}^{2+}\left(3 \mathrm{~d}^{7}\right)$ and $\Delta_{0} \sim 8500 \mathrm{~cm}^{-1}$ for $\mathrm{Ni}^{2+}\left(3 \mathrm{~d}^{8}\right)$.

Measurements of the relaxation time above $90^{\circ} \mathrm{K}$ were made by Bagguley and Griffiths (1952) and by Bleaney et al. (1951). The most important phonons will be those with energies near to $k \Theta_{D}$, where $\Theta_{D} \simeq 105^{\circ}$. For these very few of the assumptions made are valid-in particular the neglect of optical modes. Simply using an appropriate value of $\Gamma\left(x_{D}\right)$ gives a rate slower than that observed by about an order of magnitude. Further reasons for the discrepancy lie in the change in $g$ values with temperature, and the interpretation of this phenomenon by Abragam and Pryce (1950). There was evidence before starting this calculation that the Van Vleck mechanism was responsible for the relaxation, as in the dilute specimens the observed relaxation times were long, independent of concentration, and had the temperature dependence expected. The only significant feature which has not yet been confirmed in detail is the frequency dependence. This situation contrasts with that of $\mathrm{Co}^{2+}$ in $\mathrm{MgO}$ at liquid helium temperatures (Foglio and Pryce 1964) for which the $\mathrm{Co}^{2+}$ relaxation time depends strongly on the concentrations of $\mathrm{Co}^{2+}$ and other iron group impurities, and the relaxation rate is inconsistent with a calculation similar to the one described here, and with acoustic and uniaxial stress measurements. It is, of course, considerably harder to prepare impurity-free crystals of $\mathrm{MgO}$, which must be grown from the melt, than to prepare pure crystals of the water soluble Tutton salts. One feature of spin-lattice relaxation phenomena is that systems so dilute as to be essentially free from the effects of pairs or larger clusters of paramagnetic ions can be greatly influenced by low concentrations of impurities. 


\section{Acknowledgments}

It is a pleasure to acknowledge very helpful discussions with Professor M. H. L. Pryce. The work was performed while the author was in receipt of a research studentship from the Department of Scientific and Industrial Research.

\section{References}

Abragam, A., and Pryce, M. H. L., 1950, Proc. Phys. Sac. A, 63, 409.

- 1951, Proc. Roy. Soc. A, 206, 164.

Bagguley, D. M. S., and Griffiths, J. H. E., 1952, Proc. Phys. Soc. A, 65, 594.

Bleavey, B., Bowers, K. D., and Ingram, D. J. E., 1951, Proc. Phys. Soc. A, 64, 758.

Bleaney, B., Bowers, K. D., and Pryce, M. H. L., 1955, Proc. Roy. Soc. A, 228, 166.

GiLl, J. C., 1965, Proc. Phys. Soc., 85, 119.

Griffith. J. S., 1961, Theory of Transition Metal Ions (London: Cambridge University Press).

Hartree, D. R., and Hartree, W., 1936, Proc. Roy. Soc. A, 157, 490.

Hill, R. W., and Smith, P. L., 1953, Proc. Phys. Soc. A, 66, 228.

Mattuck, R. D., and Strandberg, M. W. P., 1960, Phys. Rev., 119, 1204.

Tutron, A. E. H., 1916, Phil. Trans. Roy. Soc. A, 216, 1.

VAN VleCK, J. H., 1939, J. Chem. Phys., 7, 72.

- 1940, Phys. Rev., 57, 426.

ZIMAN, J. M., 1954, Proc. Roy. Soc. A, 226, 436. 